\title{
Reasoning about Object Structures Using Ownership
}

\author{
Peter Müller \\ ETH Zurich, Switzerland \\ Peter.Mueller@inf .ethz.ch
}

\begin{abstract}
Many well-established concepts of object-oriented programming work for individual objects, but do not support object structures. The development of a verifying compiler requires enhancements of programming theory to cope with this deficiency.

In this paper, we support this position by showing that classical specification and verification techniques support invariants for individual objects whose fields are primitive values, but are unsound for invariants involving more complex object structures.

We have developed an ownership model, which allows one to structure the object store and to restrict reference passing and the operations that can be performed on references. We use this model to generalize classical object invariants to cover such object structures. We summarize the state of our work and identify open research challenges.
\end{abstract}

\section{Introduction}

Programming theory encompasses, among other fields, language semantics, program logics, and specification techniques. It provides the foundation for understanding how programs work. Together with theorem proving technology, programming theory forms the basis of program verification, in particular, of program verifiers such as the verifying compiler [17.

Programming theory has been advanced to cope with new developments in programming languages and programming practice: Interface specifications in terms of pre- and postconditions, frame axioms, and invariants describe the behavior of methods and classes [27. Abstraction functions 16 enable implementation-independent specifications of program behavior. Subtyping and dynamic method binding are addressed by behavioral subtyping [26 20]. Program logics cover a variety of programming language features [131. However, despite these achievements, programming theory still falls behind programming practice.

This position paper describes one aspect of a larger effort to advance programming theory in order to improve tool-assisted verification of realistic programs. Our work focuses on modular specification and verification of object-oriented programs. Modular verification means that a class can be verified based on its implementation and the specifications of all classes it uses, but without knowing its subclasses and clients. Modularity is a prerequisite for the scalability of 
verification techniques and tools, and for applying them to software libraries. By contrast, non-modular verification techniques require one to re-verify a class, say, a string class, in every context where it is (re-)used, which is not practical.

One of the major shortcomings of programming theory for object-oriented programming is summarized by the following position:

Many well-established concepts of object-oriented programming work for individual objects, but do not support object structures. The development of a verifying compiler requires enhancements of programming theory to cope with this deficiency.

In this paper, we support this position by discussing one particularly important concept, namely object invariants. We illustrate the problems and present ownership as a general solution to this class of problems.

For simplicity, we consider a restricted programming language here. We use a language similar to $\mathrm{C \#}$ and Java, but omit multi-threading, inheritance, and static class members. However, the presented techniques do not rely on these restrictions [18 2322 28].

Overview. In Section 2 we describe the classical technique for reasoning about invariants and its limitations. Section 3 presents a modular verification technique for invariants over object structures based on an ownership model. We summarize our progress so far and identify open research challenges in Section 4 .

\section{Classical Invariants and Their Limitations}

Invariants are predicates that specify what states of an object are consistent [16. For example, the invariant of the List class, near the top of Fig. 1] states several such properties, including that the array field is always non-null and that the array holds non-negative numbers. Thus, when calling add, for example, the expression array.length cannot cause a null pointer exception.

The invariant semantics used by classical reasoning techniques 25|26|27] is that each object has to satisfy its invariant in the pre- and poststate of each exported method. To enforce this property, classical techniques require one to prove that each exported method preserves the invariant of its receiver object. For this proof, one may assume that in the prestate of the method execution the precondition of the method holds and that all allocated objects satisfy their invariants. For constructors, one has to show that the invariant of the new object is established.

The classical techniques assume that a method can break only the invariant of its receiver object. Therefore, they are sound for invariants of individual objects whose fields are primitive values, such as points with integer coordinates. However, since they do not impose proof obligations on the invariants of other objects, these techniques do not support invariants of more complex object structures. 
Abstraction Layering is not Sound. Classical techniques do not support invariants of layers implemented on top of List. For example, the invariant of class BagWithMax in Fig. 2, which says that no element of the list is larger than a given upper bound, is generally not preserved by List's add method. That is, the classical technique's assumption that a method can break only the invariant of its receiver object is not valid for invariants that depend on the state of several objects. In particular, the call to add in BagWithMax's insert method temporarily violates the invariant of the BagWithMax object if $\mathrm{k}$ is greater than maxElem. The invariant is restored by the last statement of insert. Even if one would require add to preserve BagWithMax's invariant, this example would not be handled modularly, since modular verification of a class implies not considering its clients during its verification.

Invariants that depend on the state of objects of an underlying layer are common and important. They occur in three situations. The first is when invariants of the upper layer relate locations in the upper layer and the object states in

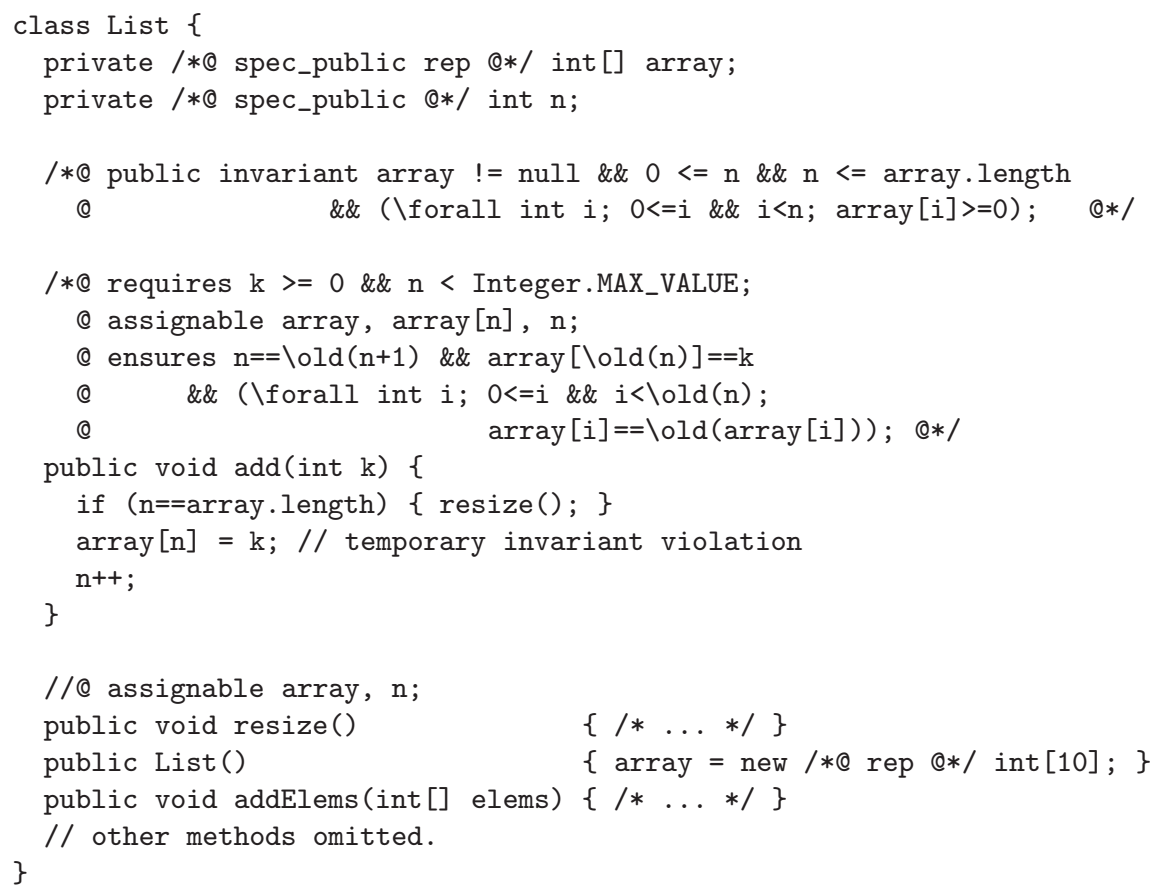

Fig. 1. Implementation and JML specification of an array-based list. Annotation comments start with an at-sign (@), and at-signs at the beginning of lines are ignored. The array object is part of the encapsulated internal representation of the list, indicated by the rep annotation. The spec_public annotation allows fields with any access modifier to be mentioned in public specifications. 


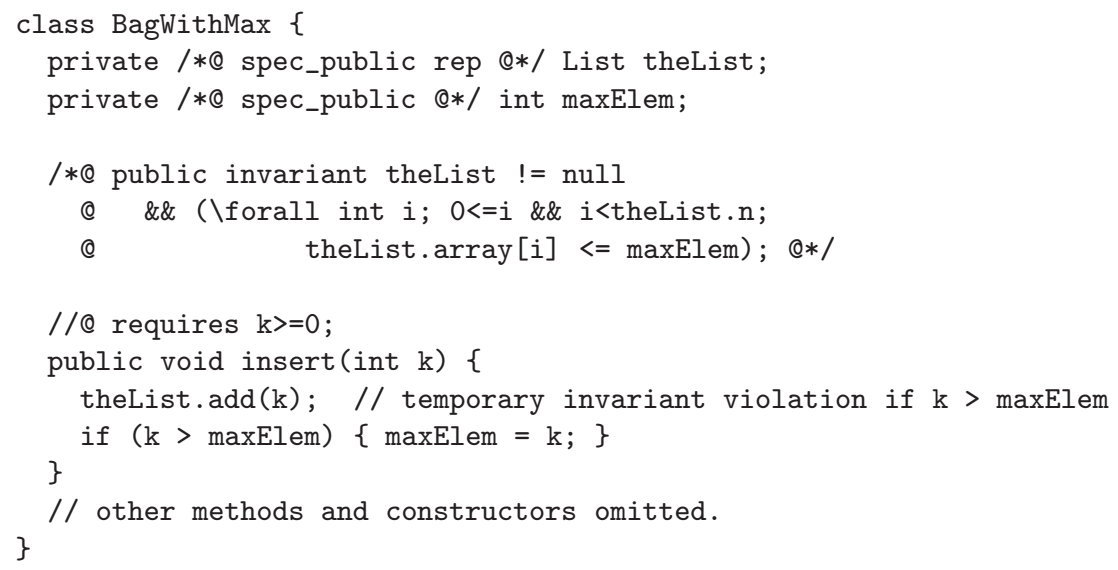

Fig. 2. Class BagWithMax builds an abstraction layer on top of List. BagWithMax's invariant depends on the state of the List object and its array.

the underlying layers, as illustrated by BagWithMax. The second is when an upper layer restricts the object states of the underlying layers. For instance, a set built on top of a list might have an invariant that excludes duplicates in the list. The third is when the invariant of an upper layer relates the states of different objects of an underlying layer. This is often the case in aggregate objects. For example, consider Family objects that aggregate different Person objects. Family's invariant could require that all Persons in a Family have the same street address.

Another way to view this soundness problem is that the classical invariant semantics is too strong for invariants over layered object structures. The class List cannot modularly know enough to establish the invariant of a class, BagWithMax, that it does not know about. Note that this problem is not due to aliasing. It occurs even if BagWithMax objects have unique references to their List objects.

Mutable Subobjects are not Sound. For example, the invariant in class List of Fig. 1 is not supported by classical techniques, because it refers to locations in the underlying array object. If a reference to the array could be exposed to other objects, then any method of the program could use such a reference to break List's invariant [10]25]. Such an alias could occur by rep exposure or by capturing as illustrated by the version of addElems in Fig. 3. With that version, the code fragment in Fig. 4 would violate List's invariant. This could happen even if the classical proof technique was used to prove the correctness of all of List's methods. This example shows that a sound technique must either restrict invariants that depend on subobjects in lower abstraction layers, or it must control aliasing and, in particular, modifications of such subobjects. 


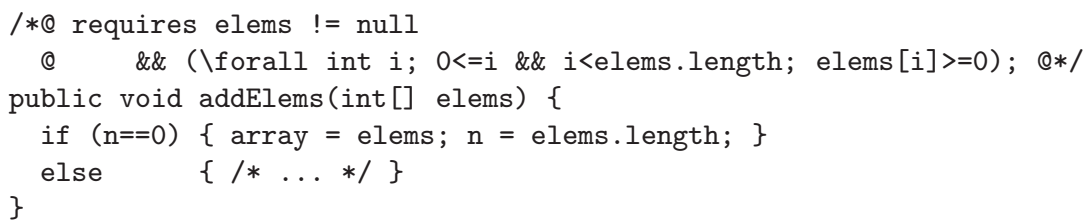

Fig. 3. A questionable implementation of the List method addElems that stores the argument array into the array field

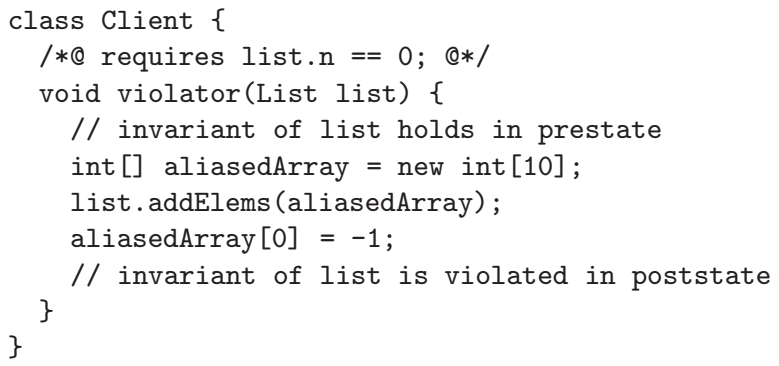

Fig. 4. Client code that shows the problem with aliased representations

\section{Ownership-Based Invariants}

Ownership allows one to structure the object store and to restrict reference passing and the operations that can be performed on references. We use ownership also to control the dependencies of invariants and to define a weaker semantics for invariants that allows layering.

\subsection{Ownership Model}

Ownership organizes objects into contexts: Each object is owned by at most one other object, called its owner. A context is the set of all objects with the same owner. The set of objects without owner is called the root context. The contexts of a program execution form a tree, where the context of all objects with owner $X$ is a child of the context containing $X$. The context tree is rooted in the root context.

Our ownership model enforces the owner-as-modifier property: All modifications of an object, $X$, must be initiated by $X$ 's owner. That is, $X$ can be referenced by any other object, but reference chains that do not pass through $X$ 's owner must not be used to modify $X$ 2228]. Therefore, owners can control modifications of owned objects. 
The ownership relation is expressed in programs by the ownership modifier rep, which can be used in field declarations and object creation expressions. In class List (Fig. 1) the rep keyword indicates that the array referenced by array and the array created in the constructor are owned by this.

Ownership and the owner-as-modifier property can be enforced by type systems or by standard verification techniques 12. For instance, our Universe type system [28] would forbid the assignment to array in Fig. 3 and require copying elems to avoid the unwanted alias.

\subsection{The Ownership Technique}

The ownership model allows one to generalize the classical technique to invariants over layered object structures. To avoid the soundness problems described in Sec. 2. we use the hierarchical structure of the ownership model to refine the classical invariant semantics.

Admissible Invariants. The ownership technique allows the invariant of an object, $X$, to depend on fields of $X$ (like the classical technique) and on fields of objects owned by $X$. The invariant of class List is an ownership-based invariant because it depends on the fields of this (array and $\mathrm{n}$ ) and on fields of the array owned by this (array. length and array[i]).

Ownership-based invariants can express properties of layered object structures. For instance, BagWithMax's invariant is allowed to depend on fields of the associated list, because the ownership model guarantees that all modifications of the list are initiated by a method of the owning BagWithMax object, and this BagWithMax method makes sure that the invariant is preserved. In particular, invariant violations through representation exposure, as illustrated in Fig. 4, are ruled out by the ownership model.

Semantics of Invariants. In Section 2, we showed that invariants over layered object structures may not hold in the pre- and poststates of all exported methods. For example, a BagWithMax object needs to temporarily violate its invariant when changing its underlying theList object. To allow such violations, we weaken the invariant semantics from the classical technique.

The weakened semantics allows a method executed on a receiver object, $X$, to violate the invariants of all objects in ancestor contexts of the context containing $X$. In particular, the method is allowed to violate the invariants of $X$ 's transitive owner objects. For instance, method add executed on a List object is allowed to violate the invariant of the owning BagWithMax object.

Ownership Proof Technique. Like the classical proof technique, the ownership proof technique requires one to prove that each exported method preserves the invariant of its receiver object. For this proof, one may assume that in the prestate of the method execution (1) the precondition of the method holds and (2) those allocated objects that are in the context of the receiver object or its descendants satisfy their invariants. However, a method must not assume that 
the (transitive) owners of the receiver object satisfy their invariants, which corresponds to the refined invariant semantics.

To illustrate how to use the ownership proof technique, consider BagWithMax's insert method. For the call to List's add method we may assume that the method preserves the invariant of theList. However, since the BagWithMax object this is the owner of the receiver of this call to add, its invariant might be broken by the call. To show that the insert method preserves this invariant, we use the postcondition of add to derive that the list after the call contains exactly the elements before the call plus the new element $\mathrm{k}$. If $\mathrm{k}$ happens to be a new maximum in the list, then BagWithMax's invariant is violated after the call, but reestablished by the subsequent assignment to maxElem. Therefore, the invariant of this is preserved.

As can be seen from the example above, responsibility for verifying invariants is divided. A method's implementor is responsible for the objects (transitively) owned by the method's receiver object, but its calling method is responsible for other objects.

This ownership proof technique is modular and sound [22 28130].

\section{Progress so Far and Open Research Challenges}

We have developed the ownership technique for object invariants in cooperation with Gary Leavens, Rustan Leino, and Arnd Poetzsch-Heffter 222830]. To handle implementations that are not supported by the ownership model such as mutually recursive data structures, we combined ownership-based invariants with so-called visibility-based invariants that gain modularity by imposing certain visibility requirements on fields 422 2430. We have also extended our methodology to static class invariants 23] and adapted it to the verification of frame properties 29]. The combination of these techniques can handle many interesting implementations.

We have developed two approaches to specifying the ownership relation and enforcing the owner-as-modifier property. The Universe type system [12]28 expresses ownership by extended type information and checks the owner-asmodifier property as part of type checking. An alternative approach 22 encodes ownership by a specification-only field that can be used in interface specifications. The owner-as-modifier property is enforced by a programming methodology that restricts how objects can be modified.

Although we have made significant progress with ownership-based verification, there are a number of open research challenges. We summarize these challenges in the following.

Ownership. Our ownership model can handle realistic applications. However, there are common implementation patterns that cannot directly be expressed, for instance, several objects sharing and modifying a common representation. We plan to generalize our ownership model to allow more implementations, in particular, multiple ownership and ownership transfer. We will also study how 
implementation patterns that are not directly supported can be rewritten to follow the ownership model.

To reduce the overhead of writing ownership annotations, we are working on ownership inference. Besides classical type inference techniques, we study runtime inference [13] to infer ownership relations. The results so far are promising. A major application of ownership inference will be to run case studies to investigate how common ownership relations are.

Specification Features. An important topic for future work is invariants over model (specification-only) fields [1921, which are useful to describe properties of data structures without referring to their concrete implementation.

History constraints [26] suffer from the same problems as the classical invariant technique when applied to object structures. We plan to extend ownership-based techniques to history constraints and to more general temporal constraints.

Specification languages like Eiffel and JML allow method calls in interface specifications. Methods that can be called in specifications must be pure, that is, side-effect free. Therefore, they can be formally modeled by mathematical functions. Especially for recursive functions, ownership can help to show that the functions are well-defined [8]. We plan to support method calls in specifications in the Boogie tool [3], which is being developed at Microsoft Research.

Automation. Practical applications of program verification require a high degree of automation. Research on automated verification has mainly focused on automated verification tools [1115] and automated theorem provers [9].

We investigate combinations of classical logic-based reasoning with extended type systems [1428], abstract interpretation [7], and static analyses. For instance, we plan to develop static, modular analyses for purity and frame properties of methods. These analyses build on the ownership structure to simplify the pointer and escape analysis used by related approaches [32].

\section{Conclusions}

We have illustrated the problems of reasoning about object structures by discussing object invariants. We have summarized the ideas of ownership-based verification, which allows one to reason about layered object structures in a modular way. Details of this approach are presented in earlier papers [2230].

Ownership allows one to structure the object store systematically. Structuring is important for many important applications. For instance, ownership has also been applied successfully to the modular verification of frame properties [29], static class invariants [23], reasoning about multi-threaded programs [5]18, confinement of internal representations of data structures [6], and proving representation independence [2]. Therefore, we believe that ownership is a fundamental principle of programming theory.

Parts of our work on the foundations of ownership and ownership-based verification have been implemented in JML 19, in the semi-automated Java Interactive Verification Environment (JIVE), and in the fully automated verification 
tool Boogie [3]. One important aspect of future work is to use these tools for non-trivial case studies.

\section{References}

1. Abadi, M., Leino, K.R.M.: A logic of object-oriented programs. In: Bidoit, M., Dauchet, M. (eds.) CAAP 1997, FASE 1997, and TAPSOFT 1997. LNCS, vol. 1214, pp. 682-696. Springer, Heidelberg (1997)

2. Banerjee, A., Naumann, D.: Representation independence, confinement, and access control. In: Principles of Programming Languages, pp. 166-177. ACM Press, New York (2002)

3. Barnett, M., Chang, B.-Y.E., DeLine, R., Jacobs, B., Leino, K.R.M.: Boogie: A modular reusable verifier for object-oriented programs. In: Submitted (2006), http://research.microsoft.com/ leino/papers/krml160.pdf

4. Barnett, M., Naumann, D.: Friends need a bit more: Maintaining invariants over shared state. In: Kozen, D. (ed.) MPC 2004. LNCS, vol. 3125, Springer, Heidelberg (2004)

5. Boyapati, C., Lee, R., Rinard, M.: Ownership types for safe programming: Preventing data races and deadlocks. In: Object-Oriented Programming, Systems, Languages, and Applications (OOPSLA), pp. 211-230. ACM Press, New York (2002)

6. Clarke, D.G., Potter, J.M., Noble, J.: Ownership types for flexible alias protection. In: Proceedings of Object-Oriented Programming Systems, Languages, and Applications (OOPSLA), October 1998. SIGPLAN Notices, vol. 33(10), ACM Press, New York (1998)

7. Cousot, P., Cousot, R.: Abstract interpretation: a unified lattice model for static analysis of programs by construction or approximation of fixpoints. In: Principles of programming languages (POPL), pp. 238-252. ACM Press, New York (1977)

8. Darvas, A., Müller, P.: Reasoning About Method Calls in Interface Specifications. Journal of Object Technology (JOT) (to appear, 2006)

9. Detlefs, D., Nelson, G., Saxe, J.B.: Simplify: A theorem prover for program checking. Technical Report HPL-2003-148, HP Labs (July 2003)

10. Detlefs, D.L., Leino, K.R.M., Nelson, G.: Wrestling with rep exposure. Research Report 156, Digital Systems Research Center (1998)

11. Detlefs, D.L., Leino, K.R.M., Nelson, G., Saxe, J.B.: Extended static checking. Research Report 159, Digital Systems Research Center (1998)

12. Dietl, W., Müller, P.: Universes: Lightweight ownership for JML. Journal of Object Technology (JOT) 4(8), 5-32 (2005)

13. Ernst, M.D., Cockrell, J., Griswold, W.G., Notkin, D.: Dynamically discovering likely program invariants to support program evolution. IEEE Transactions on Software Engineering 27(2), 1-25 (2001)

14. Fähndrich, M., Leino, K.R.M.: Declaring and checking non-null types in an objectoriented language. In: Object-oriented programing, systems, languages, and applications (OOPSLA), pp. 302-312. ACM Press, New York (2003)

15. Flanagan, C., Leino, K.R.M., Lillibridge, M., Nelson, G., Saxe, J.B., Stata, R.: Extended static checking for Java. In: Programming Language Design and Implementation (PLDI), pp. 234-245. ACM Press, New York (2002)

16. Hoare, C.A.R.: Proofs of correctness of data representation. Acta Informatica 1, 271-281 (1972) 
17. Hoare, C.A.R.: The verifying compiler: A grand challenge for computing research. Journal of the ACM 50(1), 63-69 (2003)

18. Jacobs, B., Leino, K.R.M., Schulte, W.: Verification of multithreaded objectoriented programs with invariants. In: Specification and Verification of ComponentBased Systems (SAVCBS), pp. 2-9, Technical report 04-09, Department of Computer Science, Iowa State University (2004)

19. Leavens, G.T., Baker, A.L., Ruby, C.: JML: A notation for detailed design. In: Kilov, H., Rumpe, B., Simmonds, I. (eds.) Behavioral Specifications of Businesses and Systems, pp. 175-188. Kluwer Academic Publishers, Dordrecht (1999)

20. Leavens, G.T., Weihl, W.E.: Reasoning about object-oriented programs that use subtypes (extended abstract). In: Meyrowitz, N. (ed.) OOPSLA ECOOP 1990 Proceedings. SIGPLAN, vol. 25(10), pp. 212-223. ACM Press, New York (1990)

21. Leino, K.R.M.: Toward Reliable Modular Programs. PhD thesis, California Institute of Technology (1995)

22. Leino, K.R.M., Müller, P.: Object invariants in dynamic contexts. In: Odersky, M. (ed.) ECOOP 2004. LNCS, vol. 3086, pp. 491-516. Springer, Heidelberg (2004)

23. Leino, K.R.M., Müller, P.: Modular verification of static class invariants. In: Fitzgerald, J.S., Hayes, I.J., Tarlecki, A. (eds.) FM 2005. LNCS, vol. 3582, pp. 26-42. Springer, Heidelberg (2005)

24. Leino, K.R.M., Nelson, G.: Data abstraction and information hiding. ACM Transactions on Programming Languages and Systems 24(5), 491-553 (2002)

25. Liskov, B., Guttag, J.: Abstraction and Specification in Program Development. MIT Press, Cambridge (1986)

26. Liskov, B., Wing, J.M.: A behavioral notion of subtyping. ACM Transactions on Programming Languages and Systems 16(6) (1994)

27. Meyer, B.: Object-Oriented Software Construction, 2nd edn. Prentice Hall, Englewood Cliffs (1997)

28. Müller, P.: Modular Specification and Verification of Object-Oriented Programs. LNCS, vol. 2262. Springer, Heidelberg (2002)

29. Müller, P., Poetzsch-Heffter, A., Leavens, G.T.: Modular specification of frame properties in JML. Concurrency and Computation: Practice and Experience 15, 117-154 (2003)

30. Müller, P., Poetzsch-Heffter, A., Leavens, G.T.: Modular invariants for layered object structures. In: Science of Computer Programming, ETH Zurich. Accepted for publication. Also available as TR 424 of the Department of Computer Science (2006)

31. Poetzsch-Heffter, A., Müller, P.: A programming logic for sequential Java. In: Swierstra, S.D. (ed.) ESOP 1999 and ETAPS 1999. LNCS, vol. 1576, pp. 162176. Springer, Heidelberg (1999)

32. Salcianu, A., Rinard, M.: Purity and side effect analysis for java programs. In: Cousot, R. (ed.) VMCAI 2005. LNCS, vol. 3385, pp. 199-215. Springer, Heidelberg (2005)

\section{A Discussion on Peter Müller's Presentation}

\section{Greg Nelson}

Peter, I was surprised to hear you say that you thought programmers would find it so burdensome to specify the ownership relations that it was important to do 
it by automatic inference. I would have thought that that was a small fraction of the effort in giving a functional specification for the interface, and an easy design decision to record. So, I guess that was a comment rather than a question, but I would be happy to be persuaded that you are right and I am wrong.

\section{Peter Müller}

Well, I do not think one of us is right or wrong. I think, at a certain point of the program, you make a design decision, like: the list should own the array, and somewhere record that and this should be an explicit specification. But for instance, if you use a global type system, you have to add many more types you use, to all the local variables you use, to all the parameters you use. And these additional things, which are just necessary to make type-checking work, can easily be inferred. So, I think, it is really a mixture of both.

\section{Greg Nelson}

Thank you, that actually clarifies it. But if you use the verification kinds of systems instead of the type-checking systems, then the first-step variation of ownership would be enough of an annotation?

Peter Müller: Exactly, that's enough.

\section{Rustan Leino}

Just a response also to Greg's question. Last month, I worked with one particular programmer who tried to use the ownership domains that we provide in Spec\#, and we have the ownerships there so that we can provide the invariants. I have always thought that it has been the invariants that are difficult, but in fact, this particular programmer has found great difficulties with the ownership system, which has really surprised me, and I do not think it is specific to him. But it could be that our error messages are not clear enough to explain that, but I have been surprised at that, too.

Peter Müller:(laughing) Easy question for me.

\section{Bertrand Meyer}

You are working basically with existing languages. Is there a simple language mechanism that you could propose for a new language that would make the type ownership very visible, straightforward, and under programmer control? This is somewhat in line with Greg's question. 


\section{Peter Müller}

I think, some of the ownership type systems are simple enough to actually put them in a programming language. The problem is that we still have to accept programs that do not follow these structures, because, of course, there are design patterns that do not follow the hierarchical object model. But I think there are type systems that could be actually used by mainstream programming languages. 